This document was prepared in conjunction with work accomplished under Contract No. DE-AC09-96SR18500 with the U.S. Department of Energy.

This work was prepared under an agreement with and funded by the U.S. Government. Neither the U. S. Government or its employees, nor any of its contractors, subcontractors or their employees, makes any express or implied: 1 . warranty or assumes any legal liability for the accuracy, completeness, or for the use or results of such use of any information, product, or process disclosed; or 2 . representation that such use or results of such use would not infringe privately owned rights; or 3 . endorsement or recommendation of any specifically identified commercial product, process, or service. Any views and opinions of authors expressed in this work do not necessarily state or reflect those of the United States Government, or its contractors, or subcontractors. 
WSRC-MS-2005-00638, Rev. 1

\title{
Reactivity of Resorcinol Formaldehyde Resin with Nitric Acid
}

\author{
William D. King*, Fernando F. Fondeur, William R. Wilmarth, Myra E. Pettis, and \\ Shirley W. McCollum \\ Savannah River National Laboratory, Westinghouse Savannah River Company, Aiken, \\ SC 29808 \\ *manuscript to be published in Separation Science and Technology, 2006, Issue 41
}

\begin{abstract}
Solid-state infrared spectroscopy, differential scanning calorimetry, and elemental analysis have been used to evaluate the reactivity of resorcinol formaldehyde resin with nitric acid and characterize the solid product. Two distinct reactions were identified within the temperature range $25-55^{\circ} \mathrm{C}$. The first reaction is primarily associated with resin nitration, while the second involves bulk oxidation and degradation of the polymer network leading to dissolution and off-gassing. Reaction was confirmed with nitric acid concentrations as low as $3 \mathrm{M}$ at $25^{\circ} \mathrm{C}$ applied temperature and $0.625 \mathrm{M}$ at $66{ }^{\circ} \mathrm{C}$. Although a nitrated resin product can be isolated under appropriate experimental conditions, calorimetry testing indicates no significant hazard associated with handling the dry material.
\end{abstract}

Keywords: RF, spherical resin, nitration, oxidation, IR, DSC

Received: 10/23/2005, Accepted: 3/7/2006

* author to whom all correspondence should be addressed email: william02.king@srnl.doe.gov 
WSRC-MS-2005-00638, Rev. 1

\section{INTRODUCTION}

Resorcinol formaldehyde polymers and derivatives are widely used in industrial products such as adhesives and aerogel foams, and are utilized as cation exchange materials [1]. As a result, the chemical reactivity of these materials should be of interest in a number of fields. We have characterized the reactivity of resorcinol formaldehyde (RF) ion exchange resin with nitric acid and discovered that the resin reacts with low to moderate acid concentrations and applied temperatures. This work was motivated and funded by the United States Department of Energy's Hanford River Protection Project, which focuses on the design and construction of a large scale Waste Treatment Plant (WTP) for radioactive liquids and solids currently stored in tanks at Hanford, WA. The liquid portion of the waste (supernate) is caustic and contains radioactive cesium ions (as well as other radionuclides) which must be removed prior to ultimate disposal in a glass waste form. Resorcinol formaldehyde resin is one of two ion exchange materials being considered for cesium removal in the WTP using a column processing configuration [2]. The resin is selective for cesium removal from caustic solutions even in the presence of high concentrations of sodium ions (as is the case for Hanford supernates) which compete for ion exchange sites. The cesium can be eluted from RF columns under acidic conditions and the resin can subsequently be regenerated for reuse.

For a variety of reasons, $0.5 \mathrm{M}$ nitric acid was selected for cesium elution from the WTP columns. During plant operations the potential exists for inadvertent resin exposure to nitric acid concentrations as high as $3 M$. Design controls on the columns are believed to be adequate to maintain the column temperature at $\leq 66{ }^{\circ} \mathrm{C}$ even under upset conditions, although the temperature is expected to be near $25^{\circ} \mathrm{C}$ during normal operations. 
Concerns regarding the reactivity of RF resin with nitric acid within this range of conditions motivated this work. Of particular concern were: 1) the possible formation of organonitrate species $\left(\mathrm{R}-\mathrm{NO}_{2}\right)$ with potential for detonation during spent resin storage, transfer, and disposal, and 2) the possible exposure of resin to conditions that might lead to runaway reaction within the columns resulting in rapid heat and/or pressure generation. Columns containing organic resins have been known to explode due to resin reaction and offgassing. This report describes testing conducted to identify conditions promoting RF resin reaction with nitric acid and characterize the resin after reaction.

\section{BACKGROUND}

Based on the known chemical structure of RF polymer, reaction with low to moderate nitric acid concentrations is expected. The material is composed of resorcinol $(1,3-$ dihydroxybenzene) units linked by methylene bridges. The most common structural types within the polymer are shown in Figure 1, where the methylene substituents are located in 2,4 and 4,6 ring positions [3,4]. For RF prepared under base-catalyzed conditions, structure type $\mathrm{A}$ is believed to compose $85 \%$ of the polymer repeating units. The tetra-substituted benzene rings of the $\mathrm{RF}$ structure contain four activating substituents (two hydroxyl and two alkyl) with regard to reaction with electrophilic reagents. Hydroxy substituents are known to highly activate benzene rings toward electrophilic substitution reactions [5]. For example, phenol (monohydroxybenzene) can be directly nitrated with $4.5 \mathrm{M}$ nitric acid to give a mixture of mononitrated products, while nitration of benzene occurs only in the presence of high concentrations of sulfuric 
acid. Direct nitration of polyhydroxybenzenes with nitric acid results in the formation of numerous additional products in addition to organonitrates, primarily resulting from the exothermicity of the nitration reaction and the thermal degradation of the nitrobenzene units [6].

Mono-, di-, and tri-nitroresorcinols or their metal salts are used in the explosives industry [7-11]. A number of patents have been issued for the synthesis of nitroresorcinols from nitric acid in low to moderate yields. Synthetic methods involve the addition of other reagents, such as sulfuric acid (to increase the concentration of the active electrophile, $\mathrm{NO}_{2}{ }^{+}$) or hydrogen peroxide (to decrease the concentrations of nitrogen suboxides). RF resin is also known to oxidize easily, and various oxidation products might be expected after exposure to oxidizing acids, although oxidative attack appears to be more favored by caustic conditions. Based on the scientific literature, it does not appear likely that pure nitroresorcinols can be prepared by direct reaction of RF resin with reagent grade nitric acid. Rather, a mixture of products would be expected that may include nitroresorcinols, their degradation products, and oxidized species formed by direct oxidation of the polymer.

\section{EXPERIMENTAL}

Testing was conducted on a spherical form of resorcinol formaldehyde resin prepared by a commercial vendor. The sample was received in hydrogen form under water. Prior to use, the resin was pretreated by exposure to one caustic/acid chemical cycle using $1.0 \mathrm{M}$ $\mathrm{NaOH}$ and $0.5 \mathrm{M} \mathrm{HNO}_{3}$ at ambient temperature following protocols to be used in the full- 
scale waste processing facility. Pretreated resin was stored in acid form under dilute nitric acid at $\mathrm{pH}$ 4. Resin samples for individual tests were isolated by quick vacuum filtration (2.5 to 3.0 minutes) in a disposable Nalgene ${ }^{\circledR}$ nylon filter unit. Damp test samples were collected and weighed along with duplicate small samples to be used for water content determination. Samples for water content determination were dried in a vacuum oven at $45^{\circ} \mathrm{C}$ and $-30 \mathrm{in} . \mathrm{Hg}$ until a constant dry weight was obtained. Initial test sample masses were then corrected for water content based on the values obtained for these samples.

Solutions containing various nitric acid concentrations were prepared for testing by dilution of recently purchased, concentrated nitric acid reagent. The concentration of each acid sample was verified by titration with standardized $\mathrm{NaOH}$ solution. Resin samples were contacted with acid solutions in glass vials or small polyethylene bottles. Acid contact tests were initiated by removing residual water from the resin beads with repeated washing using separate portions of nitric acid. The target acid to dry resin mass phase ratio was 20:1 (excluding the mass of the acid washes). The samples were not agitated during contact due to concerns about physical degradation of the beads. After the desired contact period, the acid was carefully decanted from the resin and the beads were quickly washed with multiple portions of deionized water and then soaked in water for several hours to remove acid from the resin pores.

Resin mass changes after nitric acid contact were determined by drying the resin samples in a vacuum oven as described above for water content determination. The final measured dry weight and the calculated initial dry weight (after correction for residual water) were used to determine mass change. Elemental analyses of selected resin 
samples were conducted by Galbraith Laboratories, Inc. of Knoxville, TN. Samples were analyzed for elemental carbon, hydrogen, nitrogen, and oxygen content using standard combustion and pyrolysis methods. All mass change tests and elemental analyses were conducted in duplicate. Resin samples used for spectroscopic characterization were not dried after acid contact, but were washed with water and stored damp.

Various analytical methods were utilized to characterize the resin samples prior to and after acid contact. Solid-state infrared (IR) spectroscopy analysis was conducted using an Attenuated Total Reflectance (ATR) diamond crystal (single reflection) situated in a "Thunderdome" accessory which had been placed in a Nicolet Nexus 670 Fourier Transform Infrared spectrometer. Collection was conducted at a resolution of $4 \mathrm{~cm}^{-1}$ using a Deuterated Triglycine Sulfate (DTGS) detector. A typical experiment involved placing 6-7 resin beads on the flat detector stage and positioning the probe near the bead surface. Each spectrum was collected using a minimum of 110 scans at a mirror speed of $0.6329 \mathrm{~cm} / \mathrm{sec}$. Differential scanning calorimetry (DSC) tests were conducted on resin samples immersed in nitric acid solutions and on neat resin after resin/nitric acid contact. DSC tests were conducted using a Perkin-Elmer PE7 calorimeter. Depending on the experiment, sealed stainless steel or unsealed aluminum DSC pans were used. A typical test sample included 5-10 $\mathrm{mg}$ of resin (dry weight basis) and approximately $30 \mathrm{mg}$ of acid. The maximum temperature for tests involving acid (typically near $95^{\circ} \mathrm{C}$ ) was limited by the nitric acid boiling point. DSC tests conducted on damp resin samples without acid involved maximum temperatures near $400^{\circ} \mathrm{C}$ and approximately $10 \mathrm{mg}$ of resin (dry weight basis). 
WSRC-MS-2005-00638, Rev. 1

\section{RESULTS AND DISCUSSION}

A spherical form of resorcinol formaldehyde resin has been observed to react with nitric acid solutions at low to moderate acid concentrations and temperatures. Clear visual evidence of reaction, as indicated by resin color change, can be seen with $3 M$ nitric acid at $25{ }^{\circ} \mathrm{C}$ applied temperature within 1 hour after acid contact. Successive resin color change, partial dissolution, and off-gassing are observed over a period of 24 hours. The rate and intensity of reaction increase with higher temperatures and acid concentrations. Nearly complete destruction of the resin results from contact with $3 \mathrm{M}$ nitric acid at $55^{\circ} \mathrm{C}$ or $8 \mathrm{M}$ acid at $25^{\circ} \mathrm{C}$ in this time period. In contrast, there is no visual evidence of significant reaction with $0.5 \mathrm{M}$ nitric acid over extended time periods at $25^{\circ} \mathrm{C}$, and chemical performance tests indicate no significant decrease in ion exchange capacity after resin exposure to these conditions. Although these results indicate that elution of columns containing RF resin with $0.5 M$ nitric acid likely does not cause significant safety hazards or degrade resin cesium removal performance, it was important to determine the threshold conditions promoting reaction. Such information could impact column temperature and acid concentration control requirements within the WTP. Furthermore, it was important to characterize the reactions of spherical RF resin with nitric acid under process extreme conditions in order to design the facility to minimize impacts of inadvertent resin exposure to off-normal conditions.

Since significant levels of nitration should lead to resin mass increases, simple mass change tests were conducted. Mass changes were determined on a dry mass basis for RF resin exposed to a range of acid concentrations and temperatures. Simple mono-nitration of each repeating polymer unit in the RF chemical structure would result in a mass 
increase of $37 \%$. For selected tests, elemental analysis of the resin samples was also conducted. Based on the known structure shown in Figure 1 (repeating unit: $\mathrm{C}_{7} \mathrm{H}_{6} \mathrm{O}_{2}$ ), asprepared RF does not contain elemental nitrogen, and the elemental content of the resin should be $68.9 \% \mathrm{C}, 5.0 \% \mathrm{H}$, and $26.2 \% \mathrm{O}$. The observation of nitrogen in resin after nitric acid contact would therefore indicate and quantify nitrogen uptake by the resin. Mono-nitration of each repeating polymer unit would result in 8.4 wt. $\% \mathrm{~N}$ and $\mathrm{C}, \mathrm{H}$, and O levels of 50.3, 3.0, and 38.3 wt. \%, respectively.

Consistent with visual observations, no mass change or nitrogen uptake was observed after 24 hour exposure of spherical RF resin to $0.5 M$ nitric acid at $25^{\circ} \mathrm{C}$ (Table 1 ). Under these conditions, no significant mass increase was observed with nitric acid concentrations as high as $2 \mathrm{M}$, indicating that the threshold acid concentration promoting reaction is $>2 \mathrm{M}$ at $25^{\circ} \mathrm{C}$. A mass increase of $24 \%$ was observed after only four hours of contact with $3 \mathrm{M}$ acid at $25^{\circ} \mathrm{C}$, which is $65 \%$ of the increase expected for mono-nitration. Interestingly, after 24 hours of contact under these conditions the mass increase had lowered to $17 \%$. Elemental analysis of this sample confirmed nitrogen uptake by the resin to give $7 \%$ nitrogen by mass (Table 1 ). Successive mass increases and decreases were consistently observed with spherical RF resin samples under various conditions promoting reaction. It is believed that the nitration reaction occurs along with independent oxidative reactions or that a portion of the organonitrate functionalities thermally decompose. Regardless, one or both of these mechanisms results in degradation of the polymer network with continued acid contact. This is consistent with visual evidence of partial polymer dissolution into the solution and off-gassing. Careful 
inspection of the elemental analysis data for this sample $(47.6 \% \mathrm{C}, 3.0 \% \mathrm{H}, 40.1 \% \mathrm{O}$, and $7.1 \% \mathrm{~N}$ ) revealed that both nitration and oxidation had occurred.

At elevated temperatures the threshold acid concentrations promoting reaction decreased (Table 1). At $55^{\circ} \mathrm{C}$ applied temperature with $3 \mathrm{M}$ nitric acid over $80 \%$ mass loss was observed within 24 hours, although for this particular experiment the acid concentration gradually increased during testing due to evaporation to near $4.5 \mathrm{M}$. Evidence of reaction was observed for $1 \mathrm{M}$ acid at $55{ }^{\circ} \mathrm{C}$ applied temperature within four hours of contact. There was no indication of reaction with $0.5 \mathrm{M}$ acid even at $66^{\circ} \mathrm{C}$ applied temperature over 24 hours. The lowest acid concentration for which evidence of nitration was observed was $0.625 \mathrm{M} \mathrm{HNO}_{3}$. As shown in Table 1, within 7 hours of exposure to this acid concentration at $66{ }^{\circ} \mathrm{C}$ (the maximum temperature believed possible within the WTP) a mass increase was observed.

Differential scanning calorimetry data for spherical RF resin immediately after contact with $3 M$ nitric acid is provided in Figure 2 (scan rate: $0.1{ }^{\circ} \mathrm{C} / \mathrm{min}$ ). A low temperature exotherm is observed soon after acid contact at an onset temperature $\leq 25^{\circ} \mathrm{C}$. The low temperature exotherm is characterized by a sharp temperature rise up to a maximum near $30^{\circ} \mathrm{C}$, followed by a gradual decrease in heat flow. Near $47^{\circ} \mathrm{C}$ under these conditions the heat flow was reduced to near the baseline. The shape of the low temperature exotherm is complex indicating that the reaction may be influenced by mass transfer of acid into the resin beads and that more than one chemical reaction may be involved. The low temperature exotherm was closely followed by a second exotherm with an onset temperature $\leq 48^{\circ} \mathrm{C}$. The second exotherm is associated with greater overall heat flow at a slower self-heating rate than was observed for the first exotherm, as indicated by a 
lower slope and larger integrated peak area. The full profile for the second exotherm could not be observed due to limitations on the maximum scan temperature associated with boiling the nitric acid reagent. Presumably the second exotherm is associated with bulk polymer oxidation and degradation resulting in destruction of the polymer network to give various dissolved and gaseous species, while the first exotherm is primarily associated with resin nitration. This is consistent with the mass change results (and visual observations) which revealed significant resin mass loss after contact with $3 \mathrm{M}$ nitric acid at $55^{\circ} \mathrm{C}$ (Table 1), but a mass increase after contact with $3 \mathrm{M}$ acid at $25^{\circ} \mathrm{C}$ applied temperature.

Surface reflectance infrared analysis of spherical RF resin beads before and after 24 hour contact with $3 M$ nitric acid revealed dramatic structural changes at the surface resulting from acid contact. The IR spectra are provided in Figure $3\left(500-2250 \mathrm{~cm}^{-1}\right.$ range only) along with the spectrum obtained by subtraction of the data obtained for the original material from that obtained after acid contact. Positive peaks in the subtraction spectrum represent new peaks formed after acid contact and negative peaks indicate missing peaks. Several prominent new peaks are observed in the range 1500 to $1800 \mathrm{~cm}^{-1}$ after acid contact. The peaks are consistent with the formation of ketone and organonitrate functionalities (see labels in Figure 3), indicating that RF resin oxidation and nitration occurred as a result of nitric acid contact [12]. Interestingly, a peak is also observed near $1630 \mathrm{~cm}^{-1}$ which is consistent with the formation of alkene functionalities. A prominent aromatic band near $1475 \mathrm{~cm}^{-1}$ is also absent in the spectrum of the material after acid contact. These observations indicate loss of aromaticity at the bead surface as a result of nitric acid contact. The IR data confirms that significant reaction occurs between 
spherical RF resin and $3 \mathrm{M}$ nitric acid at $25{ }^{\circ} \mathrm{C}$, and that the reaction is more complex than simple nitration of the benzene rings within the RF structure.

Based on the mass change, elemental analysis, and IR data it is apparent that the low temperature exotherm observed by DSC analysis was associated with nitration as well as some level of direct RF oxidation or decomposition of nitrated product to give oxidized carbon species. Additional tests were conducted on spherical RF resin which had been pre-nitrated by reaction with $3 \mathrm{MHNO}_{3}$ acid at $25{ }^{\circ} \mathrm{C}$ applied temperature. Separate DSC tests were conducted on the pre-nitrated material using $3 M$ solutions of $\mathrm{HNO}_{3}$, $\mathrm{HCl}$, and $\mathrm{NaNO}_{3}$ in order to determine whether observation of the second exotherm by DSC testing required the presence of nitric acid, or whether hydrogen or nitrate ions alone promoted reaction. As shown in Figure 4, the second exotherm is only observed in DSC scans of pre-nitrated material in the presence of nitric acid. The large decreases in heat flow observed in each of these scans between 35 and $40^{\circ} \mathrm{C}$ are associated with the sample heat capacities (a feature not observed at the slower scan speed used for the test in Figure 2). With hydrochloric acid and sodium nitrate solutions no exotherm is observed in the temperature range $55-95^{\circ} \mathrm{C}$. It therefore appears that nitrate ion and acidic conditions are required to promote bulk polymer degradation, dissolution, and off-gassing with spherical RF resin under these conditions. It is also possible that the mild levels of off-gassing and dissolution observed with $3 \mathrm{M}$ nitric acid at $25^{\circ} \mathrm{C}$ applied temperature are associated with localized heating to near $50^{\circ} \mathrm{C}$ resulting in nitric acid catalyzed thermal decomposition of a portion of the organonitrate functionalities. This would explain the difficulty in isolating a pure nitrated product. 
Since testing revealed that it is possible to isolate a nitrated (though impure) form of spherical RF resin after contact with nitric acid, it was important to evaluate the energetic properties of the nitrated material. A sample of spherical RF resin was exposed to $3 M$ nitric acid at $25^{\circ} \mathrm{C}$ for 24 hours in order to promote nitration. The presence of organonitrate functionalities in the resin was confirmed by IR after removing the acid and washing the resin with water. The nitrated material was subsequently tested as damp, neat material by DSC analysis up to $400{ }^{\circ} \mathrm{C}$ (see Figure 5). The observation of a high temperature exotherm with an onset temperature near $225^{\circ} \mathrm{C}$ is consistent with the thermal decomposition of organonitrates. The magnitude of the slope on the leading edge of the exotherm indicates rapid energetic decomposition, as would be expected for these types of materials. However, the breadth of the exotherm indicates that it is likely associated with the decomposition of a number of species with varying thermal stabilities across the temperature range 225 to $245^{\circ} \mathrm{C}$. Integration of the peak associated with the high temperature exotherm and normalization to the resin dry mass revealed that the total energetic output of the thermal decomposition reaction was only $-65 \mathrm{~J} / \mathrm{g}$ dry resin, which is well below any defined threshold for energetic compounds. It therefore appears that resin exposed to $3 M$ nitric acid does contain energetic compounds, but that the density or localized concentration is sufficiently low that the material is not considered to have significant hazard potential with regard to rapid energetic decomposition. 
WSRC-MS-2005-00638, Rev. 1

\section{CONCLUSIONS}

Visual observations and mass change, elemental analysis, and spectroscopic data revealed that the threshold conditions promoting reaction between spherical resorcinol formaldehyde resin and nitric acid over a 24 hour time scale are: $2.0-3.0 \mathrm{M}$ acid at $25^{\circ} \mathrm{C}$, 0.5-1.0 $M$ acid at $55^{\circ} \mathrm{C}$, and $0.5-0.625 M$ acid at $66^{\circ} \mathrm{C}$. The threshold conditions promoting reaction are within the range that might be experienced during Hanford Waste Treatment Plant operations, but are not expected to occur under "normal" conditions. Nitrogen incorporation into the RF chemical structure resulting from reaction with nitric acid was confirmed by elemental analysis, and IR analysis confirmed the presence of organonitrate functionalities in these samples. Careful inspection of the elemental analysis and IR data also revealed that resin oxidation or degradation of the organonitrate species had occurred as well, and a pure nitrated RF material was not isolated. DSC analysis of spherical RF resin in $3 \mathrm{M} \mathrm{HNO}_{3}$ revealed the presence of two distinct exotherms in the temperature range $25-95^{\circ} \mathrm{C}$. It appears that the first exotherm is primarily associated with nitration and partial degradation of the nitrated product, while the second exotherm is associated with bulk degradation of the polymer with significant off-gassing and dissolution. These observations regarding the reactivity of RF resin with nitric acid were not unexpected based on the known reactivities of polyhydroxy- and alkyl-substituted benzene rings. The energetic potential of the nitrated resin product was very low (as determined by DSC analysis), presumably due to the instability of the organonitrate functionalities in nitric acid, which results in thermal decomposition prior to isolation of the dry nitrated material. It should be emphasized that RF resin reactivity may differ in the actual WTP due to resin radiolysis. Based on this information, Hanford 
WSRC-MS-2005-00638, Rev. 1

WTP design personnel are reevaluating the plant ion exchange column temperature and feed acid concentration limits. 
WSRC-MS-2005-00638, Rev. 1

\section{REFERENCES}

1. Bibler, J.P.; Wallace, R.M. Cesium-specific phenolic ion exchange resin. U.S. Patent 5,441,991, August 1995.

2. Adu-Wusu, K.; Hassan, N. M.; Nash, C. A.; Marra, J. C. Removal of cesium from alkaline waste solution: Part II - Column ion exchange study. Radiochim. Acta. 2005, 93, 465 .

3. Hubler, T. L.; Franz, J. A.; Shaw, W. J.; Bryan, S. A.; Hallen, R. T.; Brown, G. N.; Bray, L. A.; Linehan, J. C. Synthesis, Structural Characterization, and Performance Evaluations of Resorcinol-Formaldehyde (R-F) Ion Exchange Resin. Pacific Northwest National Laboratory document, PNL-10744 UC-721, 1995.

4. Hubler, T. L.; Franz, J. A.; Shaw, W. J.; Hogan, M. O.; Hallen, R. T.; Brown, G. N.; Linehan, J. C. Structure/Function Studies of Resorcinol-Formaldehyde (R-F) and Phenol-Formaldehyde (P-F) Copolymer Ion Exchange Resins. Pacific Northwest National Laboratory document, PNNL-11347 UC-721, 1996.

5. Solomons, T. W. G. Organic Chemistry, $5^{\text {th }}$ Ed.; John Wiley and Sons, Inc., 1992.

6. Schmitt, R. J.; Ross, D. S.; Wolfe, J. F. Process for Preparing 4,6-Dinitroresorcinol. U.S. Patent 4,745,232, May 1988.

7. Kostanecki, S. V.; Feinstein, B. Chemische Berichte. 1888, 21, 3119.

8. Typke, P. G. W. Chemische Berichte. 1883, 16, 551.

9. Finch, A.; Payne, J. Thermochemistry of Nitroresorcinols, Part 2. 2,4- and 4,6Dinitroresorcinol. Thermochimica Acta. 1991, 189, 109.

10. Turker, L. PM3 Treatment of Lead Styphnate and its Mono-ionic Forms. J. Molecular Structure (Theochem). 2004, 681, 143.

11. Kruper, W. J.; Lysenko, Z.; Hull, Jr., J. W.; Frycek, G. J. One-Step Preparation of 4,6-Dinitroresorcinol from Resorcinol. U.S. Patent 5,371,303, December, 1994.

12. Pretsch, E.; Bühlmann, P.; Affolter, C. Structure Determination of Organic Compounds, $3^{\text {rd }}$ Ed; ; Springer: New York, 2000. 
Table 1-1. Mass Change and Elemental Analysis Results After Spherical RF Resin Contact with Nitric Acid under Various Conditions.

\begin{tabular}{|c|c|c|}
\hline $\begin{array}{l}\text { Initial Acid Conc. } \\
\qquad(M)^{1}\end{array}$ & $\begin{array}{l}\text { \% Mass Change } \\
\text { (dry basis) }^{2}\end{array}$ & $\begin{array}{l}\text { Weight \% Nitrogen } \\
\text { (dry basis) }\end{array}$ \\
\hline \multicolumn{3}{|c|}{$25^{\circ} \mathrm{C}$ applied temperature } \\
\hline 0.5 & $+0.3(1.2)$ & $<0.5$ \\
\hline 2.0 & $+0.8(0.7)$ & $<0.5$ \\
\hline $3.0(4 \mathrm{hr})$ & $+23.6(0.7)$ & --- \\
\hline 3.0 & $+16.9(1.2)$ & $7.11(0.06)$ \\
\hline \multicolumn{3}{|c|}{$55^{\circ} \mathrm{C}$ applied temperature } \\
\hline 0.5 & $-1.1(0.7)$ & --- \\
\hline $1.0(4 \mathrm{hr})$ & $+19.1(0.3)$ & --- \\
\hline $1.0^{3}$ & $+2.7(1.5)$ & --- \\
\hline $3.0^{3}$ & $-80.2(0.7)$ & --- \\
\hline \multicolumn{3}{|c|}{$66^{\circ} \mathrm{C}$ applied temperature } \\
\hline 0.5 & $-1.1(0.5)$ & --- \\
\hline $0.625(7 \mathrm{hr})$ & $+5.0(1.3)$ & --- \\
\hline $0.75(5 \mathrm{hr})$ & $+7.1(0.1)$ & --- \\
\hline
\end{tabular}

${ }^{1} 24$ hour contact time unless otherwise indicated 2 average of duplicate results, standard deviation in parentheses ${ }^{3}$ evaporation resulted in 50\% increase in acid concentration during testing for these samples 


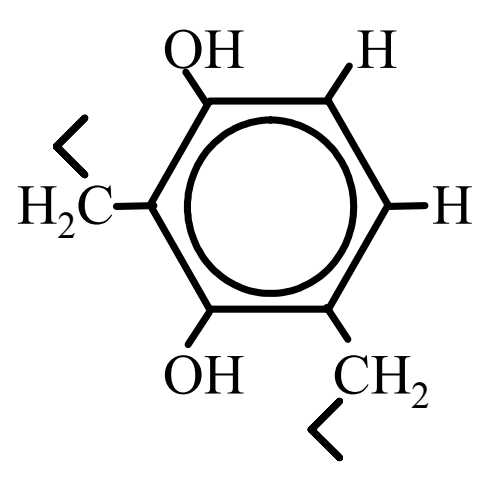

Type A

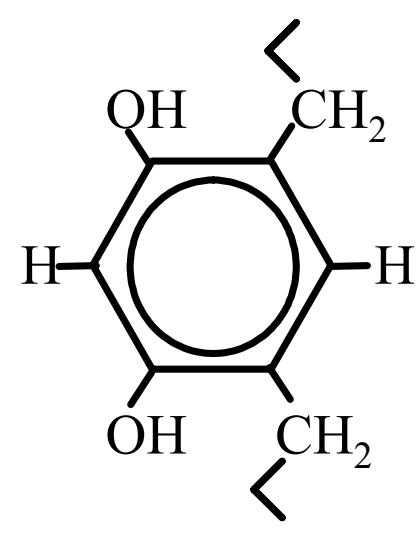

$\underline{\text { Type B }}$

Figure 1. Primary structural types within resorcinol formaldehyde polymer. 
WSRC-MS-2005-00638, Rev. 1

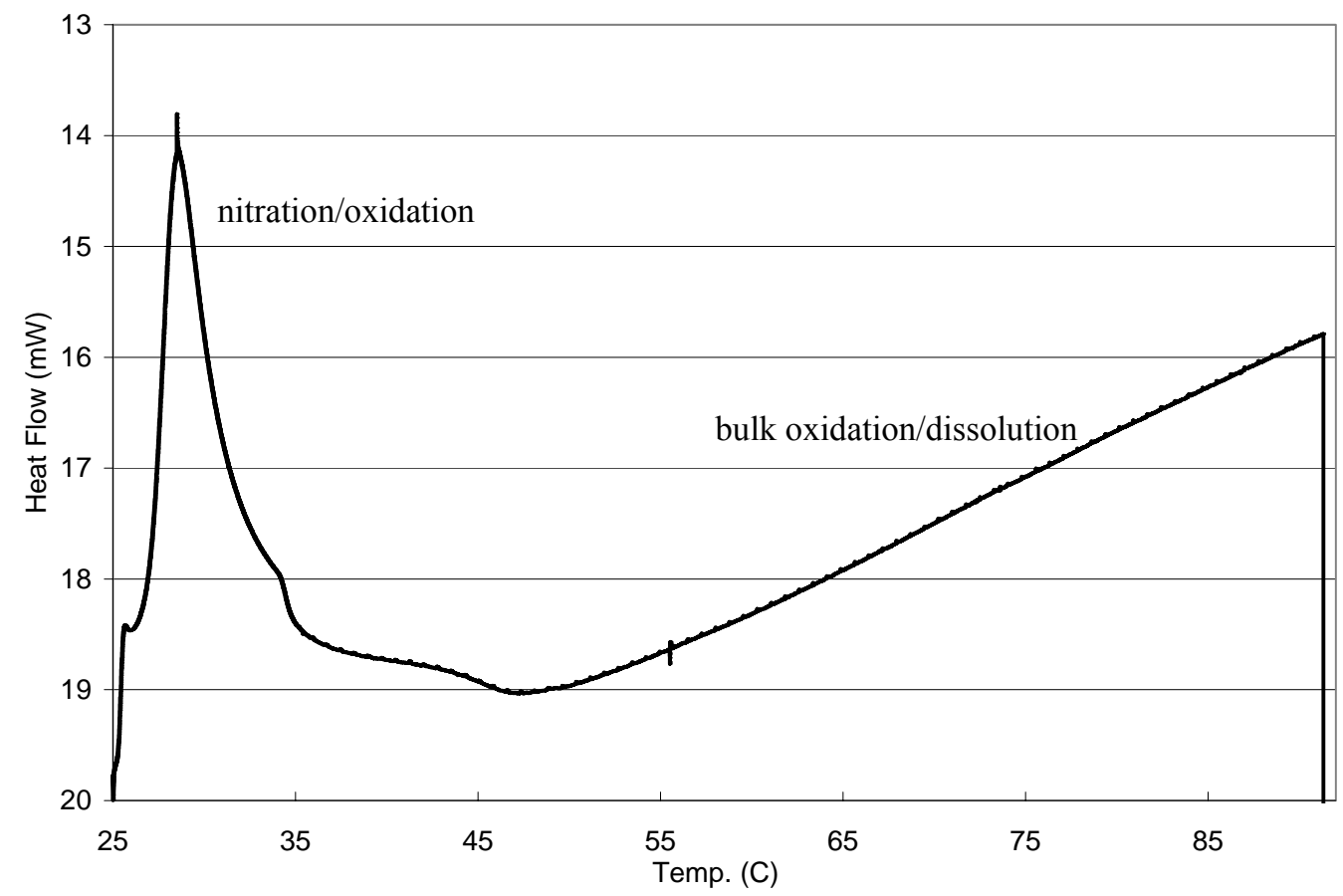

Figure 2. Differential scanning calorimetry data for spherical RF resin in $3 M$ nitric acid (scan rate: $0.1{ }^{\circ} \mathrm{C} / \mathrm{min}$ ). 
WSRC-MS-2005-00638, Rev. 1

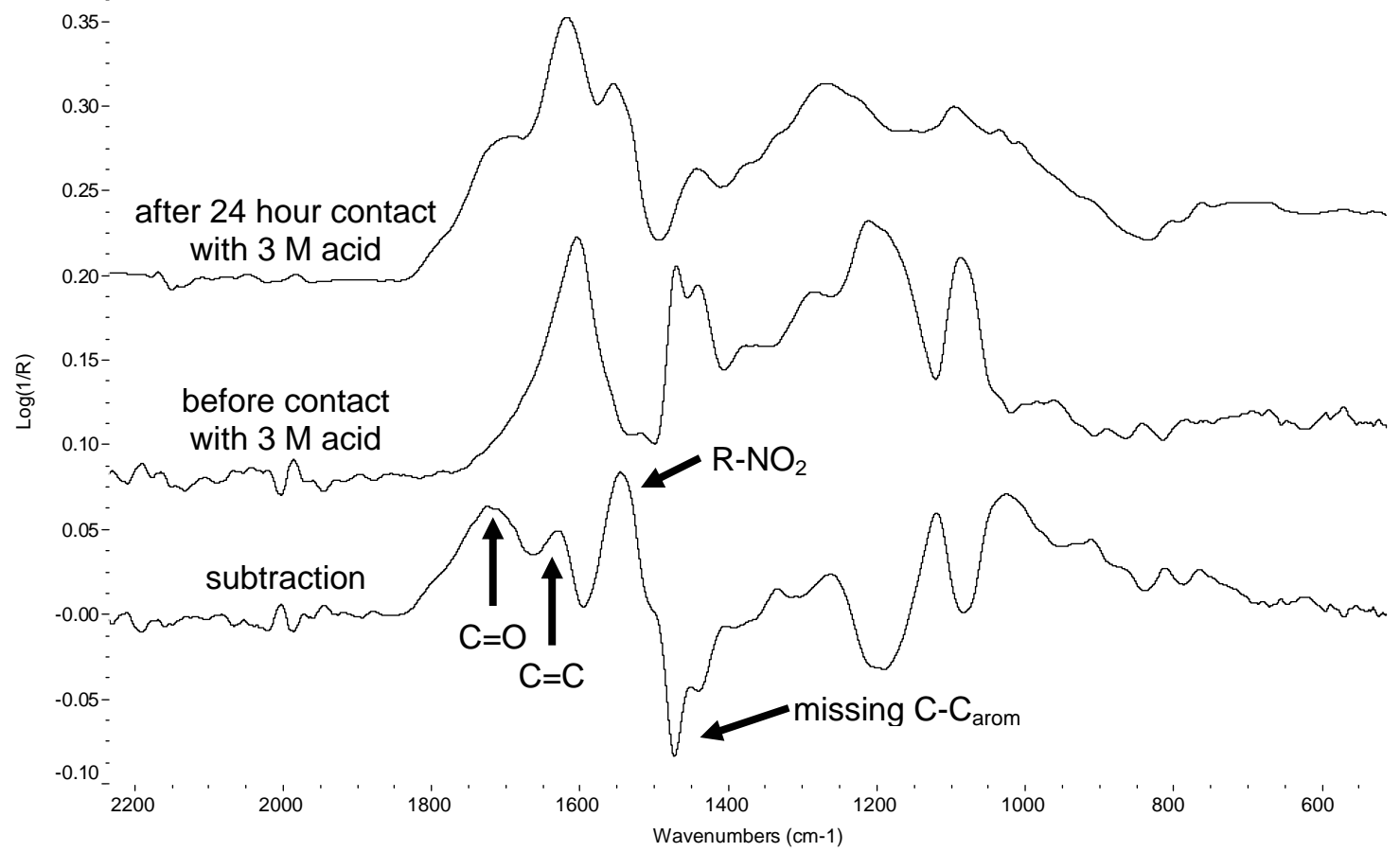

Figure 3. Surface reflectance infrared spectra of spherical RF resin before and after 3 $M$ nitric acid exposure at $25^{\circ} \mathrm{C}$ (lower trace generated by subtraction of center spectrum from upper spectrum). 
WSRC-MS-2005-00638, Rev. 1

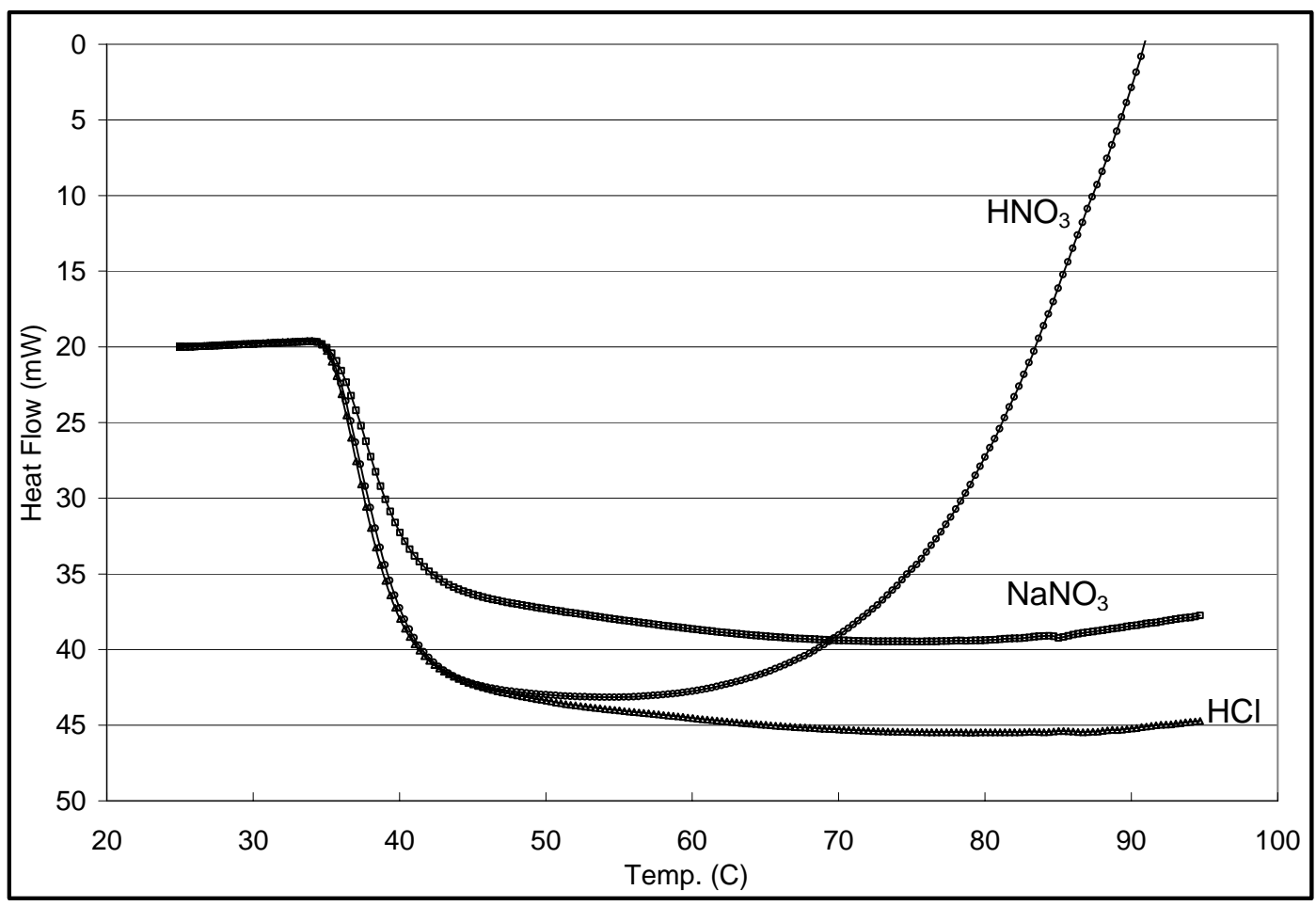

Figure 4. DSC scans of pre-nitrated/oxidized spherical $\mathrm{RF}$ resin in $3 \mathrm{M}$ nitric acid, sodium nitrate, and hydrochloric acid solutions (scan rate: $10^{\circ} \mathrm{C} / \mathrm{min}$ ). 
WSRC-MS-2005-00638, Rev. 1

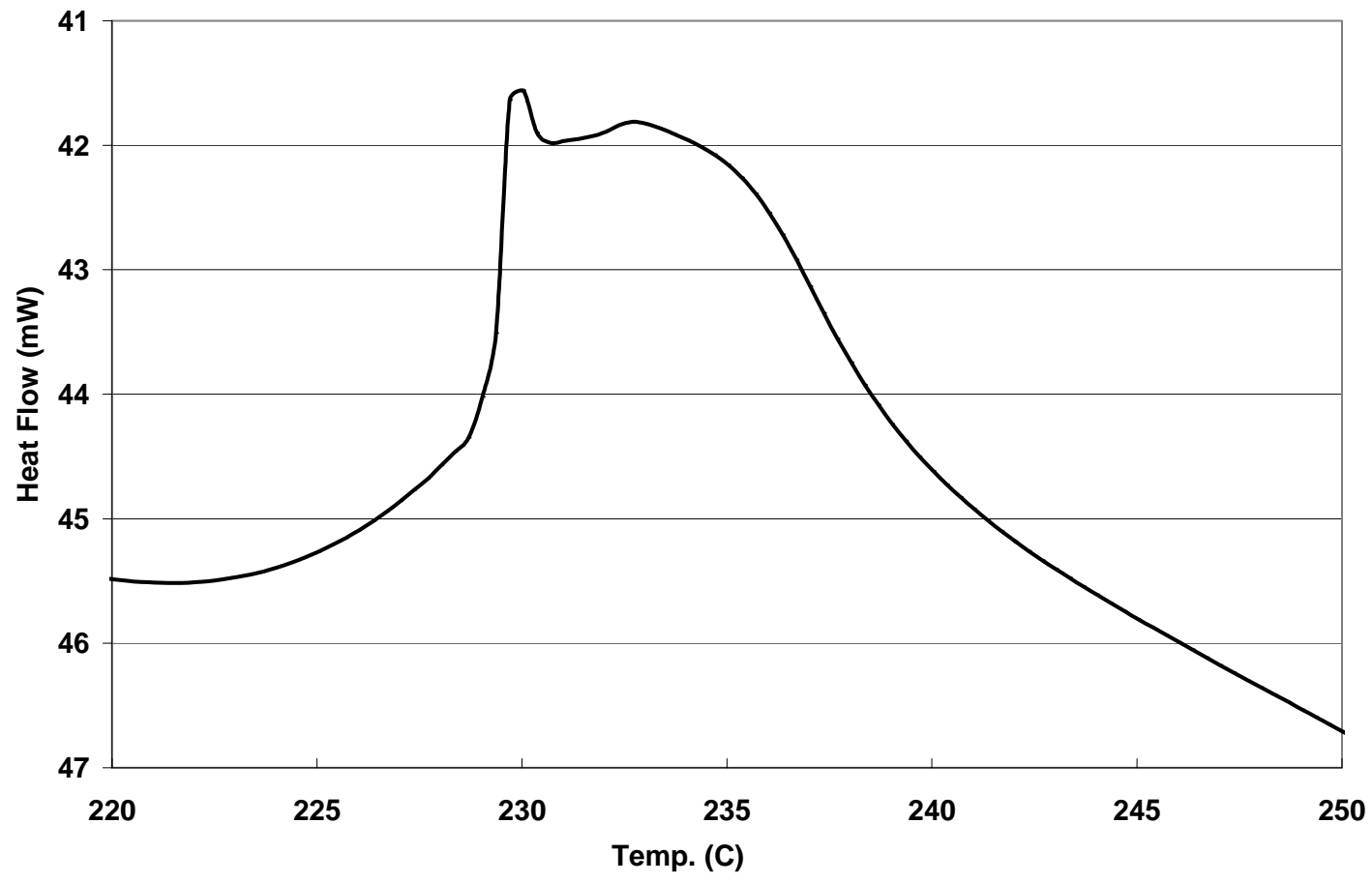

Figure 5. High temperature DSC scan of damp, spherical RF resin after contact with $3 \mathrm{M} \mathrm{HNO}_{3}$ acid at $25^{\circ} \mathrm{C}$ for 24 hours (scan rate: $10^{\circ} \mathrm{C} / \mathrm{min}$ ). 